Gazi University
Journal of Science
http://dergipark.gov.tr/gujs

\title{
Homotopy Perturbation Elzaki Transform Method for Obtaining the Approximate Solutions of the Random Partial Differential Equations
}

\author{
Halil ANAC ${ }^{1, *}$ (D) Mehmet MERDAN $^{1}$, ${ }^{(1)}$ Tulay KESEMEN ${ }^{2}$ (D) \\ ${ }^{I}$ Department of Mathematical Engineering, Gumushane University, 29100, Gumushane, Turkey \\ ${ }^{2}$ Department of Mathematics, Karadeniz Technical University, 61080, Trabzon, Turkey
}

\section{Highlights}

- The series solution is focused.

- Homotopy Perturbation Elzaki Transform Method is used.

- The moments are obtained.

\begin{tabular}{l} 
Article Info \\
\hline \\
Received: 23 Sep 2020 \\
Accepted: 22 Sep 2021 \\
Keywords \\
\hline Expected value \\
Homotopy perturbation \\
elzaki transform \\
method, \\
Variance
\end{tabular}

\section{INTRODUCTION}

The ordinary differential equations (ODEs) that include random constants or random variables have been current important matters that are named as random ordinary differential equations (RODEs). The topic of random partial differential equations (RPDEs) have been used to analyze a lot of applications such as engineering, mathematical biology and physics problems. Recently, a lot of scientific problems which belongs to fluid mechanics, control theory, climate models, physics and so on are solved. The random evolution partial differential equations have been examined. Also, they have analyzed the heat and Schrödinger equations by random parameters [1]. A new solution approach for these RPDEs based on deep learning has been given [2]. The numerical approximation of RPDEs have been examined [3]. The mean square approach and Laplace transform method have been used to solve random hyperbolic PDEs [4]. The new Sumudu transform iterative method is applied to obtain the approximate solutions of RPDEs [5].

There are extremely few papers about random nonlinear partial differential equations (RNPDEs) in the literature. The partial differential equations (PDEs) that include are random variables or stochastic processes are defined as random partial differential equations. RPDEs are very important in a lot of applications in a lot of scientific areas. It is often impossible to analytically solve RNPDEs. Thus, a lot of numerical methods for RPDEs, ODEs and PDEs have been established. There are a lot of numerical methods such as Adomian decomposition method (ADM) [6], homotopy perturbation method (HPM) [710], differential transform method (DTM) [11-16], variational iteration method (VIM) [17], finite difference method [18-19], random finite difference scheme [20,21] and a lot of methods. 
This work studies RNPDEs and to acquire the approximate solutions of these equations by the HPETM. Jena and Chakraverty have applied this method to solve Navier-Stokes equation of fractional order [22].

The main motivation of this work is to obtain the approximate solutions of the RNPDEs by the homotopy perturbation Elzaki transform method and calculate the first and second moments of these approximate solutions. Besides, the graphs of these moments have been plotted in MAPLE software.

\section{BASIC DEFINITIONS}

A few basic definitions and features are given in this section.

Definition 2.1 [23] Let the the function $f(x)$ is probability density function for random variable $X$. Then, $X$ gets normal distribution and is named the normal random variable

$f(x)=\frac{1}{\sigma \sqrt{2 \pi}} e^{-\frac{1}{2}\left(\frac{x-\mu}{\sigma}\right)^{2}},-\infty<x<\infty,-\infty<\mu<\infty, \sigma^{2}>0$.

If $X$ has the normal distribution by parameters $\mu$ and $\sigma^{2}$, the first and second moments of $X$ are defined by

$$
E(X)=\mu, \operatorname{Var}(X)=\sigma^{2} .
$$

Definition 2.2 [23] Let the the function $f(x)$ is probability density function for random variable $X$. Then, $X$ gets uniform distribution and is named the uniform random variable

$f(x)=\frac{1}{\beta-\alpha}, \quad \alpha<x<\beta$.

If $X$ has the uniform distribution by parameters $\alpha$ and $\beta$, the first and second moments of $X$ are defined by

$E(X)=\alpha, \operatorname{Var}(X)=\beta$

Definition 2.3 [24] The Elzaki transform (ET) of the function $g(t)$ for $t>0$ is defined by

$E\{g(t)\}=T(v)=v \int_{0}^{\infty} g(t) e^{-\frac{t}{v}} d t$.

Definition 2.4 [24] The Elzaki transforms of the partial derivatives are as follows.

$E\left[\frac{\partial g(x, t)}{\partial t}\right]=\frac{1}{v} T(x, v)-v g(x, 0), E\left[\frac{\partial^{2} g(x, t)}{\partial t^{2}}\right]=\frac{1}{v^{2}} T(x, v)-g(x, 0)-v \frac{\partial g(x, 0)}{\partial t}$,

$E\left[\frac{\partial g(x, t)}{\partial x}\right]=\frac{d}{d x}[T(x, v)], E\left[\frac{\partial^{2} g(x, t)}{\partial x^{2}}\right]=\frac{d^{2}}{d x^{2}}[T(x, v)]$.

Also, the normal and uniform distributions are used in the examples.

\section{A HYBRID METHOD}

Consider the NPDE by the initial conditions 
$\left\{\begin{array}{c}D w(u, t)+L w(u, t)+N w(u, t)=g(u, t) \\ w(u, 0)=h(u), w_{v}(u, 0)=f(u)\end{array}\right.$

where $D$ is the second order linear differential operator, $L, N$ are respectively linear and nonlinear differential operators and also, the order of $L$ is smaller than two, $g(u, t)$ is the nonhomogeneous term [25].

If ET is applied to both sides of (7), then it is obtained as [25]

$E[D w(u, t)]+E[L w(u, t)]+E[N w(u, t)]=E[g(u, t)]$.

If the differential property of Elzaki transform and initial conditions (ICs) are used, then (9) is obtained.

$E[w(u, t)]=v^{2} E[g(u, t)]+v^{2} h(u)+v^{3} f(u)-v^{2} E[L w(u, t)+N w(u, t)]$.

If the inverse Elzaki transform is applied to both sides of (9), then it is found as [25]

$w(u, t)=G(u, t)-E^{-1}\left\{v^{2} E[L w(u, t)+N w(u, t)]\right\}$

where $G(u, t)$ is the term that arises by the nonhomogeneous term and ICs.

Now, HPM

$w(u, t)=\sum_{n=0}^{\infty} p^{n} w_{n}(u, t)$

is applied and the nonlinear term has been decomposed as

$N w(u, v)=\sum_{n=0}^{\infty} p^{n} H_{n}(w)$

where $H_{n}(w)$ are given by

$H_{n}\left(w_{0}, w_{1}, \ldots, w_{n}\right)=\frac{1}{n !} \frac{\partial}{\partial p^{n}}\left[N\left(\sum_{i=0}^{\infty} p^{i} w_{i}\right)\right]_{p=0}, n=0,1,2, \ldots$

(11) and (12) are substituted in (10), it is obtained as [25]

$\sum_{n=0}^{\infty} p^{n} w_{n}(u, t)=G(u, t)-p\left\{E^{-1}\left\{v^{2} E\left[L \sum_{n=0}^{\infty} p^{n} w_{n}(u, t)+\sum_{n=0}^{\infty} p^{n} H_{n}(w)\right]\right\}\right\}$

This is the coupled of the ET and HPM.

If the same powers of $p$ are compared, then the iterations which obtained are as follows:

$p^{0}: w_{0}(u, t)=G(u, t)$

$p^{1}: w_{1}(u, t)=-E^{-1}\left\{v^{2} E\left[L w_{0}(u, t)+H_{0}(w)\right]\right\}$,

$p^{2}: w_{2}(u, t)=-E^{-1}\left\{v^{2} E\left[R w_{1}(u, t)+H_{1}(w)\right]\right\}$,

$p^{3}: w_{3}(u, t)=-E^{-1}\left\{v^{2} E\left[R w_{2}(u, t)+H_{2}(w)\right]\right\}$

$\vdots$ 
Thus, the series solution of (7) is given as [25]

$u(x, t)=\sum_{i=0}^{\infty} u_{i}(x, t)$

\section{NUMERICAL EXPERIMENTS}

Example 4.1. Consider the RNPDE,

$$
\left\{\begin{array}{c}
w_{t}(u, t)+w(u, t) w_{u}(u, t)=-B u+2 A^{2} u^{3}-3 A B u^{2}+B^{2} u t^{2} \\
u(x, 0)=A u^{2}
\end{array}\right.
$$

where $A$ is random variable which has Normal distribution by parameters $\alpha=3$ and $\beta=2$ and $B$ is random variable which has uniform distribution by parameters $\alpha=2, \beta=1$, i.e. $A \sim N(\alpha=3, \beta=2), B \sim U(\alpha=$ $2, \beta=1)$.

If ET is implemented to (16) and the differential property of ET is used, then (17) is obtained as

$E[w(u, t)]=A u^{2} v^{2}-B u v^{3}+2 A^{2} u^{3} v^{3}-3 A B u^{2} v^{4}+2 B^{2} u v^{5}-v E\left[w(u, t) w_{u}(u, t)\right]$

If the inverse ET is applied to (17), then (18) is obtained as

$w(u, t)=A u^{2}-B u t+2 A^{2} u^{3} t-\frac{3 A B u^{2} t^{2}}{2}+\frac{B^{2} u t^{3}}{3}-E^{-1}\left\{v E\left[w(u, t) w_{u}(u, t)\right]\right\}$.

Now, the HPM is applied, then it is obtained as

$\sum_{n=0}^{\infty} p^{n} w_{n}(u, t)=u^{2}-B u t+2 A^{2} u^{3} t-\frac{3 A B u^{2} t^{2}}{2}+\frac{B^{2} u t^{3}}{3}-p\left[E^{-1}\left\{v E\left[\sum_{n=0}^{\infty} p^{n} H_{n}(w)\right]\right\}\right]$

where $H_{n}(w)$ are He's polynomials which show nonlinear terms.

Some components of $H_{n}(u)$ are as follows:

$H_{0}(w)=w_{0} w_{0 u}$,

$H_{1}(w)=w_{0} w_{1 u}+w_{1} w_{0 u}$,

$H_{2}(w)=w_{0} w_{2 u}+w_{1} w_{1 u}+w_{2} w_{0 u}$,

$\vdots$

If the same powers of $p$ are compared, then they are obtained as

$$
\begin{aligned}
& p^{0}: w_{0}(u, t)=A u^{2}-B u t+2 A^{2} u^{3} t-\frac{3 A B u^{2} t^{2}}{2}+\frac{B^{2} u t^{3}}{3} \\
& H_{0}(w)=\left(A u^{2}-B u t+2 A^{2} u^{3} t-\frac{3 A B u^{2} t^{2}}{2}+\frac{B^{2} u t^{3}}{3}\right)\left(2 A u-B t+6 A^{2} u^{2} t-3 A B u t^{2}+\frac{B^{2} t^{3}}{3}\right) \\
& p^{1}: w_{1}(u, t)=-2 A^{2} u^{3} t+\frac{A B u^{2} t^{2}}{2}-3 A^{3} u t^{2}+3 A^{2} B u^{3} t^{3}-\frac{10 A B^{2} u^{2} t^{4}}{3}+A B u^{2} t^{2}-\frac{B^{2} u t^{3}}{3} \\
& +\frac{B^{3} u t^{5}}{15}-2 A^{3} u^{4} t^{2}+A^{2} B u^{3} t^{3}-4 A^{4} u^{5} t^{3}+\frac{13 A^{3} B u^{4} t^{4}}{2}-\frac{2 A^{2} B^{2} u^{3} t^{5}}{15}-\frac{A B^{2} u^{2} t^{4}}{8}+\frac{3 A^{3} B u^{4} t^{4}}{4}
\end{aligned}
$$


$-\frac{4 A^{2} B^{2} u^{3} t^{5}}{5}+\frac{A B^{3} u^{2} t^{6}}{12}+\frac{2 A^{2} B u^{3} t^{3}}{3}-\frac{5 A B^{2} u^{2} t^{4}}{12}+\frac{3 A^{3} B u^{4} t^{4}}{2}-\frac{A^{2} B^{2} u^{2} t^{5}}{5}+\frac{B^{3} u t^{5}}{3}-\frac{2 A^{2} B^{2} u^{3} t^{5}}{5}$ $+\frac{A B^{3} u^{2} t^{6}}{18}+80 A B^{3} u^{2} t^{6}-80 B^{4} u t^{7}$

Therefore, the series solution of (16) has been obtained as

$$
\begin{aligned}
& w(u, t)=w_{0}(u, t)+w_{1}(u, t)=A u^{2}-B u t+2 A^{2} u^{3} t-\frac{3 A B u^{2} t^{2}}{2}+\frac{B^{2} u t^{3}}{3}-2 A^{2} u^{3} t+\frac{A B u^{2} t^{2}}{2} \\
& -3 A^{3} u t^{2}+3 A^{2} B u^{3} t^{3}-\frac{10 A B^{2} u^{2} t^{4}}{3}+A B u^{2} t^{2}-\frac{B^{2} u t^{3}}{3}+\frac{B^{3} u t^{5}}{15}-2 A^{3} u^{4} t^{2}+A^{2} B u^{3} t^{3}-4 A^{4} u^{5} t^{3} \\
& +\frac{13 A^{3} B u^{4} t^{4}}{2}-\frac{2 A^{2} B^{2} u^{3} t^{5}}{15}-\frac{A B^{2} u^{2} t^{4}}{8}+\frac{3 A^{3} B u^{4} t^{4}}{4}-\frac{4 A^{2} B^{2} u^{3} t^{5}}{5}+\frac{A B^{3} u^{2} t^{6}}{12}+\frac{2 A^{2} B u^{3} t^{3}}{3} \\
& -\frac{5 A B^{2} u^{2} t^{4}}{12}+\frac{3 A^{3} B u^{4} t^{4}}{2}-\frac{A^{2} B^{2} u^{2} t^{5}}{5}+\frac{B^{3} u t^{5}}{3}-\frac{2 A^{2} B^{2} u^{3} t^{5}}{5}+\frac{A B^{3} u^{2} t^{6}}{18} \\
& +80 A B^{3} u^{2} t^{6}-80 B^{4} u t^{7} .
\end{aligned}
$$

Let $A \sim N(\alpha=3, \beta=2)$ and $B \sim U(\alpha=2, \beta=1)$.

So the approximated values of $A, A^{2}, A^{3}, A^{4}, A^{5}, A^{6}, A^{7}, A^{8}, B, B^{2}, B^{3}, B^{4}$ are obtained as follows.

$$
\begin{aligned}
& E(A)=3, E\left(A^{2}\right)=13, E\left(A^{3}\right)=63, E\left(A^{4}\right)=345, E\left(A^{5}\right)=1323, E\left(A^{6}\right)=13029, \\
& E\left(A^{7}\right)=88119, E\left(A^{8}\right)=622608, E(B)=\frac{3}{2}, E\left(B^{2}\right)=\frac{7}{3}, E\left(B^{3}\right)=\frac{15}{4}, E\left(B^{4}\right)=\frac{31}{5} .
\end{aligned}
$$

The first moment of (20) has been obtained as

$$
\begin{aligned}
& E[w(u, t)]=3 u^{2}-\frac{3 u t}{2}+26 u^{3} t-\frac{9 u^{2} t^{2}}{4}-189 u t^{2}-\frac{39 u^{3} t^{3}}{2}-\frac{147 u^{2} t^{4}}{6}+\frac{u t^{5}}{2}-126 u^{4} t^{2} \\
& +\frac{65}{2} u^{3} t^{3}-1380 u^{5} t^{3}+\frac{189 u^{4} t^{4}}{4}+567 u^{4} t^{4}-\frac{728 u^{3} t^{5}}{45}-\frac{7 u^{2} t^{4}}{8}+\frac{1701 u^{4} t^{4}}{8}+\frac{5 u^{2} t^{6}}{16}-\frac{7 u^{2} t^{4}}{4} \\
& -\frac{91 u^{2} t^{5}}{15}-\frac{182 u^{3} t^{5}}{15}+\frac{5 u^{2} t^{6}}{4}+900 u^{2} t^{6}-496 u t^{7}
\end{aligned}
$$

The first moment of (20) is shown in Figure 1. 


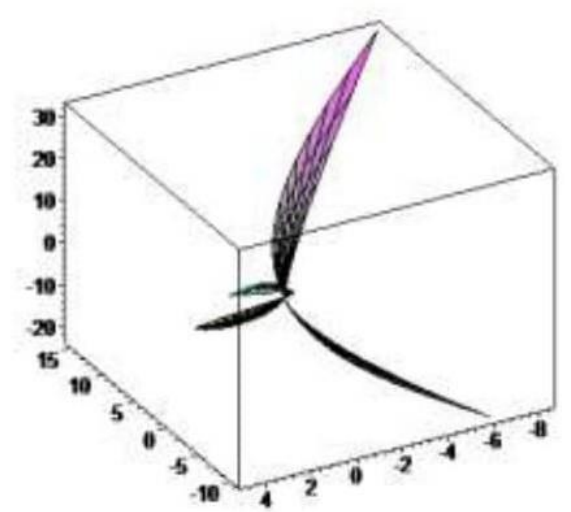

Figure 1. Time-dependent variation of first moment of (20)

Let $A \sim N(\alpha=3, \beta=2)$ and $B \sim U(\alpha=2, \beta=1)$. Then the variance of (20) is calculated by MAPLE software.

The second moment of (20) is shown in Figure 2.

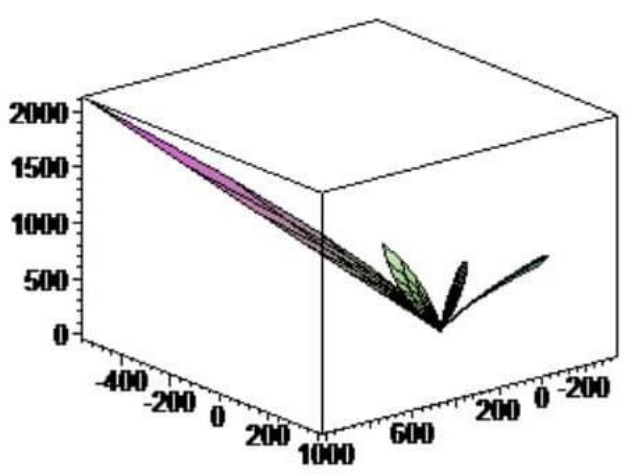

Figure 2. Time-dependent change of second moment for (20)

Example 4.2. Consider the RNPDE,

$\left\{\begin{array}{c}w_{t}(u, t)+w(u, t) w_{u}(u, t)=A B t^{3}+B^{2} u t^{2}+2 A t+B u, \\ w(u, 0)=-B u\end{array}\right.$

where $A$ is random variable which has Normal distribution by parameters $\alpha=2$ and $\beta=1$ and $B$ is random variable which has uniform distribution by parameters $\alpha=2, \beta=3$, i.e. $A \sim N(\alpha=2, \beta=1), B \sim U(\alpha=$ $2, \beta=3)$.

If ET is implemented to (21) and the differential property of ET is used, then (22) is obtained as

$E[w(u, t)]=6 A B v^{6}+2 B^{2} u v^{5}+2 A v^{4}+B u v^{3}-B u v^{2}-v E\left[w(u, t) w_{u}(u, t)\right]$.

If the inverse ET is applied to (22), then (23) is obtained as

$w(u, t)=\frac{A B t^{4}}{4}+\frac{B^{2} u t^{3}}{3}+A t^{2}+B u t-B u-E^{-1}\left\{v E\left[w w(u, t) w_{u}(u, t)\right]\right\}$.

Also, HPM is applied, it is obtained as 
$\sum_{n=0}^{\infty} p^{n} w_{n}(u, t)=\frac{A B t^{4}}{4}+\frac{B^{2} u t^{3}}{3}+A t^{2}+B u t-B u-p\left[E^{-1}\left\{v E\left[\sum_{n=0}^{\infty} p^{n} H_{n}(w)\right]\right\}\right]$

where $H_{n}(w)$ are He's polynomials which show nonlinear terms.

Some components of $H_{n}(w)$ are as follows:

$H_{0}(w)=w_{0} w_{0 u}$

$H_{1}(w)=w_{0} w_{1 u}+w_{1} w_{0 u}$

$H_{2}(w)=w_{0} w_{2 u}+w_{1} w_{1 u}+w_{2} w_{0 u}$

If the same powers of $p$ are compared, then they are obtained as

$p^{0}: w_{0}(u, t)=\frac{A B t^{4}}{4}+\frac{B^{2} u t^{3}}{3}+A t^{2}+B u t-B u$,

$H_{0}(w)=\left(\frac{A B t^{4}}{4}+\frac{B^{2} u t^{3}}{3}+A t^{2}+B u t-B u\right)\left(\frac{B^{2} t^{3}}{3}+B t-B\right)$

$p^{1}: w_{1}(u, t)=\frac{A B^{3} t^{8}}{96}+\frac{7 A B^{2} t^{6}}{72}-\frac{A B^{2} t^{5}}{20}+\frac{B^{4} u t^{7}}{63}+\frac{2 B^{3} u t^{5}}{15}-\frac{B^{3} u t^{4}}{6}+\frac{A B t^{4}}{4}-\frac{A B t^{3}}{3}+\frac{B^{2} u t^{3}}{3}$

$-B^{2} u t^{2}+B^{2} u t$

Therefore, the series solution of (21) is acquired as

$$
\begin{aligned}
& w(u, t)=w_{0}(u, t)+w_{1}(u, t)=\frac{A B t^{4}}{2}+\frac{2 B^{2} u t^{3}}{3}+A t^{2}+B u t-B u+\frac{A B^{3} t^{8}}{96}+\frac{7 A B^{2} t^{6}}{72}-\frac{A B^{2} t^{5}}{20} \\
& +\frac{B^{4} u t^{7}}{63}+\frac{2 B^{3} u t^{5}}{15}-\frac{B^{3} u t^{4}}{6}-\frac{A B t^{3}}{3}-B^{2} u t^{2}+B^{2} u t
\end{aligned}
$$

Let $A \sim N(\alpha=2, \beta=1)$ and $B \sim U(\alpha=2, \beta=3)$.

So the approximated values of $A, A^{2}, B, B^{2}, B^{3}, B^{4}, B^{5}, B^{6}, B^{7}, B^{8}$ are obtained as follows.

$$
\begin{aligned}
& E(A)=2, E\left(A^{2}\right)=5, E(B)=\frac{5}{2}, E\left(B^{2}\right)=\frac{19}{3}, E\left(B^{3}\right)=\frac{65}{4}, E\left(B^{4}\right)=\frac{211}{5}, E\left(B^{5}\right)=\frac{665}{6} \\
& E\left(B^{6}\right)=\frac{2059}{7}, E\left(B^{7}\right)=\frac{6305}{8}, E\left(B^{8}\right)=\frac{19171}{9} .
\end{aligned}
$$

The first moment of (25) is calculated as

$$
\begin{aligned}
& E[w(u, t)]=\frac{5 t^{4}}{2}+\frac{38 u t^{3}}{9}+2 t^{2}+\frac{53 u t}{6}-\frac{5 u}{2}+\frac{65 t^{8}}{192}+\frac{133 t^{6}}{108}-\frac{19 t^{5}}{30}+\frac{211 u t^{7}}{315}+\frac{13 u t^{5}}{6}-\frac{65 u t^{4}}{24} \\
& -\frac{5 t^{3}}{3}-\frac{19 u t^{2}}{3} .
\end{aligned}
$$

The first moment of (25) is shown in Figure 3. 


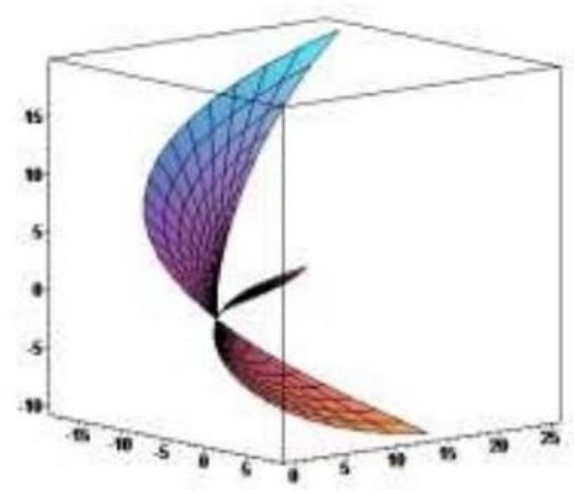

Figure 3. Time-dependent variation of first moment of (25)

For $A \sim N(\alpha=2, \beta=1)$ and $B \sim U(\alpha=2, \beta=3)$ the variance of (25) is calculated by MAPLE software.

The second moment of (25) is shown in Figure 4.

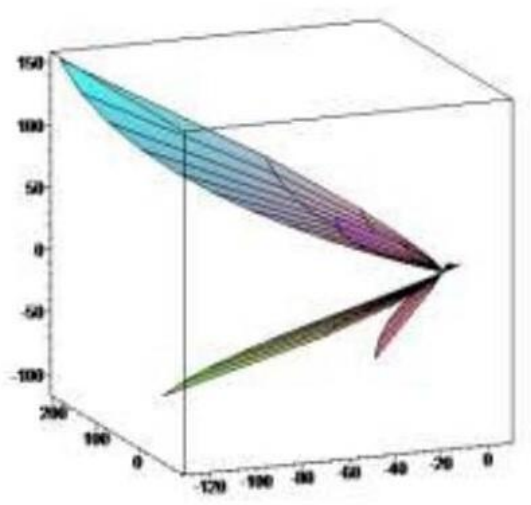

Figure 4. Time-dependent variation of second moment of (25)

\section{RESULTS AND DISCUSSIONS}

We plotted the graphs of the first and second moments of the temperature $w(u, t)$ of HPETM solutions for examples 4.1 and 4.2. Figure 1 shows the first moment results obtained by using HPETM for example 4.1. Besides, Figure 2 shows the second moment results obtained by using HPETM for example 4.1. It is observed from Figures 1-2 that these moments of the temperature $w(u, t)$ decreases when the values of space variable $u$ increases and time $t$ is constant. Also, we have inferred from Figures 1-2 that these moments of the temperature $w(u, t)$ decreases when the values of space variable $u$ is constant and time $t$ increases. Figure 3 shows the first moment results obtained by using HPETM for example 4.2. Figure 4 shows the second moment results obtained by using HPETM for example 4.2. It is observed from Figures 3-4 that these moments of the temperature $w(u, t)$ increases when the values of space variable $u$ and time $t$ increase.

\section{CONCLUSION}

In this paper, RNPDEs are analyzed by HPETM. Besides, the functions for the first and second moments of the approximate analytical solutions of the RNPDEs have been acquired by using both normal and uniform distributions. We have observed that the whole structures of the surface graphs that are acquired in Maple software differ for Example 4.1. In the same way, we have deduced that the whole structures of the surface graphs that are acquired in Maple software differ for Example 4.2. Also, the approximate 
analytical solutions of the RNPDEs have been quickly and successfully obtained by HPETM. Therefore, HPETM is quickly, efficient and superior in obtaining the approximate analytical solutions of RNPDEs.

\section{CONFLICTS OF INTEREST}

No conflict of interest was declared by the authors.

\section{REFERENCES}

[1] Lü, Q., Zuazua, E., "Averaged controllability for random evolution partial differential equations", Journal de Mathématiques Pures et Appliquées, 105(3): 367-414, (2016).

[2] Nabian, M.A., Meidani, H., "A deep learning solution approach for high-dimensional random differential equations", Probabilistic Engineering Mechanics, 57: 14-25, (2019).

[3] Guignard, D., "Partial differential equations with random input data: A perturbation approach", Archives of Computational Methods in Engineering, 26(5): 1313-1377, (2019).

[4] Egorova, V.N., Jódar, L., "Quadrature Integration Techniques for Random Hyperbolic PDE Problems", Mathematics, 9(2): 160, (2021).

[5] Anaç, H., Merdan, M., and Kesemen, T., "Solving for the random component time-fractional partial differential equations with the new Sumudu transform iterative method", SN Applied Sciences, 2: 1$11,(2020)$.

[6] Khudair, A. R., Ameen, A.A., and Khalaf, S.L., "Mean square solutions of second-order random differential equations by using adomian decomposition method", Applied Mathematical Sciences, 5: 2521-2535, (2011).

[7] He, J.H., "Homotopy perturbation method: a new nonlinear analytical technique", Applied Mathematics and Computation, 135(1): 73-79, (2003).

[8] He, J.H., "Homotopy perturbation method for solving boundary value problems", Physics Letters A, 350(1-2): 87-88, (2006).

[9] He, J.H., "Addendum: new interpretation of homotopy perturbation method", International Journal of Modern Physics B, 20(18): 2561-2568, (2006).

[10] Odibat, Z., Momani, S., "Modified homotopy perturbation method: application to quadratic Riccati differential equation of fractional order", Chaos, Solitons \& Fractals, 36(1): 167-174, (2008).

[11] Anaç, H., Merdan, M., Bekiryazıc1, Z., and Kesemen, T., "Bazı Rastgele Kısmi Diferansiyel Denklemlerin Diferansiyel Dönüşüm Metodu ve Laplace-Padé Metodu Kullanarak Çözümü”, Gümüşhane Üniversitesi Fen Bilimleri Enstitüsü Dergisi, 9(1): 108-118, (2019).

[12] Ayaz, F., "Solutions of the system of differential equations by differential transform method", Applied Mathematics and Computation, 147(2): 547-567, (2004).

[13] Kangalgil, F., Ayaz, F., "Solitary wave solutions for the KdV and $\mathrm{mKdV}$ equations by differential transform method", Chaos, Solitons \& Fractals, 41(1): 464-472, (2009).

[14] Merdan, M., "A new application of modified differential transformation method for modeling the pollution of a system of lakes", Selçuk Journal of Applied Mathematics, 11(2): 27-40, (2010). 
[15] Yüzbaşi, Ş., Ismailov, N., "Differential Transform Method to Solve Two-Dimensional Volterra Integral Equations with Proportional Delays", New Trends in Mathematical Sciences, 5(4): 65-71, (2017).

[16] Zhou, J.K. "Differential Transform and Its Applications for Electrical Circuits", China: Huazhong University Press, (1986).

[17] He, J.H., "Variational iteration method-a kind of non-linear analytical technique: some examples", International Journal of Non-Linear Mechanics, 34(4): 699-708, (1999).

[18] Ekolin, G., "Finite difference methods for a nonlocal boundary value problem for the heat equation", BIT, 31: 245-261, (1991).

[19] Smith, G.D. "Numerical Solution of Partial Differential Equations", UK: Oxford University Press, (1965).

[20] Cortés, J.C., Jódar, L., Villafuerte, L., and Villanueva, R.J., "Computing mean square approximations of random diffusion models with source term", Mathematics and Computers in Simulation, 76(1-3): 44-48, (2007).

[21] El-Tawil, M.A., Sohaly, M.A., "Mean square convergent three points finite difference scheme for random partial differential equations", Journal of the Egyptian Mathematical Society, 20(3): 188204, (2012).

[22] Jena, R.M., Chakraverty, S., "Solving time-fractional Navier-Stokes equations using homotopy perturbation Elzaki transform", SN Applied Sciences, 1(1): 1-13, (2019).

[23] Feller, W. "An introduction to probability theory and its applications", USA: John Wiley \& Sons, (1971).

[24] Elzaki, T.M., "Applications of new transform "Elzaki transform" to partial differential equations", Global Journal of Pure and Applied Mathematics, 7(1): 65-70, (2011).

[25] Elzaki, T.M., Hilal, E.M.A., "Homotopy perturbation and Elzaki transform for solving nonlinear partial differential equations", Mathematical Theory and Modeling, 2(3): 33-42, (2012). 